Quiconque travaille sur un campus universitaire n'a pu que constater le nombre croissant d'étudiants en provenance du continent asiatique. On a ainsi vu arriver depuis une dizaine d'années de plus en plus d'étudiants chinois dans toutes les disciplines, scientifiques et littéraires, y compris les sciences physiques. Cela ne fait que traduire l'émergence rapide en science et technologies des pays asiatiques, de la Chine à l'Inde, de Taïwan à Singapour. C'est un fait majeur de notre horizon scientifique, comme on peut le constater avec la présence grandissante d'auteurs asiatiques dans les conférences internationales et les journaux spécialisés.

\title{
Les relations Est-Ouest à l'échelle du continent asiatique
}

Les Européens ont mis quelque temps à se rendre compte de l'avance prise par les Américains et à réagir pour ne pas leur laisser le champ libre. On s'est par exemple aperçu que le débit des liaisons Internet entre Amérique du Nord et Asie était tellement rapide que toutes les liaisons Europe-Asie, au lieu d'être directes, transitaient par les USA... ce qui indiquait l'intensité de la coopération déjà en cours. En sciences physiques, des membres d'agences de recherche européennes (comme le CNRS) et asiatiques (comme le JSPS au Japon) s'en sont alarmés et ont lancé l'an dernier une initiative pour réfléchir à la coopération entre les deux continents, et renforcer les moyens institutionnels y afférant. Ayant sollicité le concours de la Société Européenne de Physique (EPS) et de l'Association of Asia-Pacific Physical Societies (AAPPS), ils ont proposé d'organiser un sommet Asie-Europe en physique, Asia Europe Physics Summit ASEPS. La première réunion plénière de ce sommet s'est tenue en mars dernier à Tsukuba (Japon), pour discuter du développement de coopérations multilatérales à l'échelle du continent ${ }^{(1)}$. La présence forte d'agences européennes (comme le CNRS, avec plusieurs directeurs d'institut présents) et asiatiques, la signature d'un protocole d'accord entre EPS et AAPPS à cette occasion, sont autant de signes clairs de la volonté d'avancer. La position de la Commission Européenne devra être précisée. Cependant, les discussions à Tsukuba ont surtout été dominées par les échanges sur les grands instruments et grandes infrastructures de recherche, internationaux par nature (et relevant souvent de la physique nucléaire et de la physique des particules). La collaboration en réseau entre équipes de recherche travaillant dans d'autres domaines (physique atomique, matière condensée, information quantique, etc.) n’a pas été abordée sur le fond et devra être incluse dans l'avenir.

Un domaine de coopération clé pour assurer la mobilité des jeunes asiatiques vers l'Europe, et à peine effleuré lors de la rencontre de Tsukuba, est celui de la formation scientifique. Comment fournir une formation de haut niveau en sciences physiques, dispensée sur place de façon à atteindre les chercheurs débutants dans leur environnement naturel ? Certaines institutions européennes (comme l'Imperial College) ont déjà compris l'importance de cet aspect, et ont passé des accords de coopération avec des universités asiatiques. Les pays européens, et en particulier la France, ont des atouts majeurs à faire valoir dans ce type de coopération, avec les prestigieuses Écoles d'été de Physique établies depuis longtemps en Europe. L'an dernier, durant le mois de juillet 2009, a été organisée à Singapour une session d'été de I'École des Houches (la première hors d'Europe) sur " gaz ultra-froids et information quantique », avec des enseignants venus de France, $d^{\prime}$ Europe et d'Asie. Cette école a été un grand succès, rassemblant une soixantaine d'étudiants dont plus de la moitié en provenance $d^{\prime} A s i e^{(2)}$. La suite donnée à cette expérience $d^{\prime}$ un genre nouveau sera un test de l'ouverture à l'international de la formation scientifique de haut niveau, et de l'absence de frilosité des institutions françaises.

Finalement, un enjeu à plus long terme concerne les publications scientifiques. On peut constater une présence accrue d'articles de groupes asiatiques dans les revues européennes et américaines. Le niveau scientifique de ces articles s'améliore régulièrement. La survie des journaux scientifiques européens passera peut-être par des accords de partenariat avec leurs correspondants asiatiques. Tout cela devrait être inclus dans l'initiative Asie-Europe en cours.

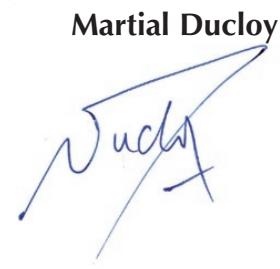

